\title{
Research on Acceleration Data Recovery System Based on the Process of High-Speed Projectile Entering Water Impact
}

\section{Zhang Shuai}

Beijing Institute of Technology https://orcid.org/0000-0003-3614-1609

shao zhiyu ( $\nabla$ shaozhiyu@bit.edu.cn )

Beijing Institute of Technology

\section{Original Article}

Keywords: Data collection and recovery, real-time cache, experomental verification, disconnection trigger

Posted Date: February 3rd, 2021

DOI: https://doi.org/10.21203/rs.3.rs-162435/v1

License: (c) (i) This work is licensed under a Creative Commons Attribution 4.0 International License.

Read Full License 


\section{Title page}

\section{Research on Acceleration Data Recovery System Based on the Process of High-speed Projectile Entering Water Impact}

Shuai Zhang, born in 1996, is currently a Master candidate at State Key Laboratory of Explosion Science and Technology, Beijing Institute of Technology, China. His research interests include Mechatronics, mechanical testing.

Tel: 17801206564; E-mail: 2569119978@qq.com

Zhi-Yu Shao, born in 1977, is currently an engineer at, State Key Laboratory of Explosion Science and Technology, Beijing Institute of Technology, China. He received his PhD degree from Beijing University of Aeronautics and Astronautics, China, in 2007. His research interests include mechachonics engineering, man-machine system, robotics and ocean engineering.

Tel: 010-68911579; E-mail: shaozhiyu@bit.edu.cn

Corresponding author: Zhi-Yu Shao E-mail: shaozhiyu@bit.edu.cn 


\title{
Research on Acceleration Data Recovery System Based on the Process of High-speed Projectile Entering Water Impact
}

\author{
Shuai Zhang • Zhi-Yu Shao
}

Received January 22, 2021; revised February xx, 201x; accepted March xx, 201x

(c) Chinese Mechanical Engineering Society and Springer-Verlag Berlin Heidelberg 2017

\begin{abstract}
In the high-speed water-entry shock experiment, we need to store and recover the acceleration data generated during the water-entry shock process. In response to this need, we designed a two-dimensional acceleration measurement combined device system. The system uses the IEPE sensor as the sensitive element, and de-signs an efficient constant current source circuit and a low-power signal conditioning circuit. Use high-precision A/D (ADS1256) converter to achieve analog-to-digital conversion, the data communication be-tween the main control unit and ADC is realized through the serial peripheral interface SPI, and high-speed data transmission is completed. The main control unit stores the collected digital signals in the corresponding memory card through a new polling storage technology. The experimental results show that the system adopts the disconnection trigger method to effectively avoid the false trigger phenomenon during testing. The polling storage technology of real-time cache solves the problem of partial data loss caused by power outage in a specific water impact environment. By comparing the waveform energy difference with the data obtained by dynamic simulation and verifying by mathematical analysis, it can be known that there is no frame error or frame loss in the transmission data. The data recovery device can meet the conditions for high-speed acceleration data recovery, and can provide reliable basic data support for water impact related research.
\end{abstract}

Keywords: Data collection and recovery - real-time cache • experimental verification $\bullet$ disconnection trigger

Zhi-Yu Shao

shaozhiyu@bit.edu.cn

State Key Laboratory of Explosion Science and Technology, Beijing Institute of Technology, Beijing, 100081, China

\section{Introduction}

In the high-speed water impact test, we need to collect data on the acceleration generated during the impact. This process includes real-time data collection, data storage and subsequent data processing. Due to the inherent characteristics of the projectile specimen material, in actual experiments, the test piece is often broken and the recovery facility is exposed to water. So, we need to design a new data recovery device.

Looking at the relevant literature, most scholars use MEMS acceleration, but from Golovinskiy's paper, we know that MEMS has too few experimental data for measurement performance under a large range [1], Therefore, in the high overload problem, more and more scholars use IEPE sensors. Levinzon F and FANG Y Y improved the IEPE sensors. After improving the sensitive originals of the amplifying circuit, it per-forms well in large range [2-3]; But we need to design the constant current source and signal conditioning module by ourselves.

And ZHOU R Q designs the signal conditioning circuit and DC bias circuit based on the research of missile body recovery, At the same time, in order to avoid accidental touch, a new disconnection + internal trigger scheme is designed, the system uses double buffering for data collection. In order to facilitate disassembly, the system separates the measurement module and the acquisition module [4]. However, the use of an active filter will cause energy loss in the signal in the passband, and the load effect will be more obvious, which is not conducive to data analysis in water related fields.

In the part of data acquisition, LIAN Shu ren et al. designed an FPGA-based missile recovery system based on research related to missile penetration. Select a specific 
chip and design a signal amplifier circuit. At the same time, the above circuit is stepped down to make it consistent with the A/D module range. Use the double buffer method to measure the muzzle acceleration curve [5]. LONG Z J, CUI Q, ZHANG T's paper introduced the specific bus communication process and the implementation process and advantages of double buffering, but it cannot solve the problem of data loss caused by power failure after a crash [6-8]. The paper by WANG K, WU Z H, et al. introduced the content of buffer protection and overall design when the overall package is packaged. [9-10] But it does not involve the introduction of specific high-speed data recovery hardware.

Aiming at the demonstration and analysis, we design an efficient constant current source circuit and signal conditioning circuit. The system adopts a new type of A/D converter and main control unit. The system designs a polling cache method to solve the problem of data loss caused by power failure. The data recovery device can meet the conditions for the recovery of high-speed acceleration data, and can provide reliable basic data support for water impact related research.

\section{Material and Methods}

In order to obtain the acceleration generated when the gun is shot into the water impact, an acceleration data recovery system under a high impact state is used. The device can be known by numerical simulation; the maximum acceleration is $3.9 \times 10^{4}$, and an acceleration is designed for this requirement. The data recovery system, which is installed inside the impact projectile, is mainly composed of a power supply module, an acceleration sensor module, a data acquisition storage module, and a data return module. The system structure diagram is shown in Figure 1 :

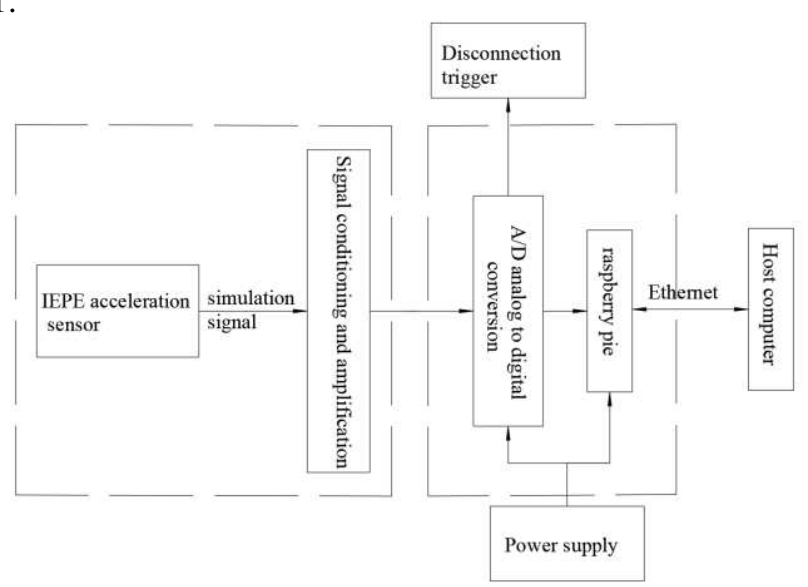

Figure 1 System structure block diagram

The power supply module selects the lithium battery provided by the Raspberry $\mathrm{Pi}$ and connects to the charging port. The Raspberry Pi 4B does not have a power supply switch. Weld the DS-01-1P DIP switch on the power supply end of the Raspberry Pi. After the switch is closed, it can be the Raspberry Pie provides a continuous and stable current supply.

The acceleration sensor module is responsible for providing a stable, anti-interference analog signal to the core controller. The IEPE sensor with high anti-noise is adopted, and the supporting signal conditioning circuit is designed.

The digital-to-analog converter in the data acquisition storage module uses an 8-channel 24-bit high-precision ADC (4-channel differential input) produced by Texas Instrument. It also has a 30ksps sampling rate and is compatible with a $5 \mathrm{~V}$ range SPI serial interface.

The Raspberry Pi 4B is selected as the core controller for the data acquisition and storage module. The Raspberry $\mathrm{Pi}$ 4B is a SOC composed of a quad-core BCM2711, which is a 64 -bit $1.5 \mathrm{Ghz}$ chip based on a $28 \mathrm{~nm}$ process. Using the built-in WiringPi library, you can configure the basic peripheral interface driver, use SPI to transmit the digital information transmitted by the A/D module, use embedded $\mathrm{C}$ for programming, and the Raspberry $\mathrm{Pi} 4 \mathrm{~B}$ and ADS1256 communicate through the SPI bus. To ensure the accuracy and real-time of the data. The external trigger method is adopted, the physical shock characteristics at the moment of initiation are used, and the disconnection trigger method is adopted to facilitate the reliable acquisition of acceleration data fragments during the acceleration process of the water entering.

The data return module uses the Raspberry Pi 4B Gigabit Ethernet interface, and stores the stored acceleration data to the upper computer through the upper computer interactive software, and imports the corresponding data processing software for subsequent data analysis.

\subsection{Hardware design}

\subsubsection{Acceleration sensor module}

The function of the acceleration sensor module is to provide a stable, anti-interference analog signal. The piezoelectric acceleration sensor uses materials with piezoelectric effect as the pressure-sensitive element, which has the characteristics of wide frequency response range, high sensitivity, good dynamic characteristics, and strong anti-interference ability. It meets the recycling requirements of this test, so the CA-YD-180 series acceleration sensor of Jiangsu Lian-Neng Electronic Technology Co., Ltd. is selected. The main technical indicators of the sensor are shown in Table 1. 
Table 1 Main technical indicators of CA-YD-180

\begin{tabular}{ccc}
\hline Number & Parameter & value \\
\hline 1 & Reference sensitivity & $0.106 \mathrm{mV} / \mathrm{m}^{-2}$ \\
2 & Frequency Range & $1--12000 \mathrm{~Hz}$ \\
3 & Maximum lateral sensitivity ratio & $<5 \%$ \\
4 & Maximum allowable acceleration & $5 \times 10^{4} \mathrm{~m} \cdot \mathrm{s}^{-2}$ \\
5 & Installation thread & $\mathrm{M} 5$ \\
6 & weight & $9 \mathrm{~g}$ \\
\hline
\end{tabular}

CA-YD-180 series sensors need 2-10 mA constant current source to supply power. This article compares the pros and cons of many kinds of constant current source circuits. Finally, considering the product accura-cy requirements, product cost, and circuit board size in the actual design, the constant current source circuit based on the LM334 chip is used to draw the PCB board. Using the circuit schematic shown in Figure 2 to supply power to the acceleration sensor, the constant current value generated by the LM3334 at this time is $8.9 \mathrm{~mA}$.

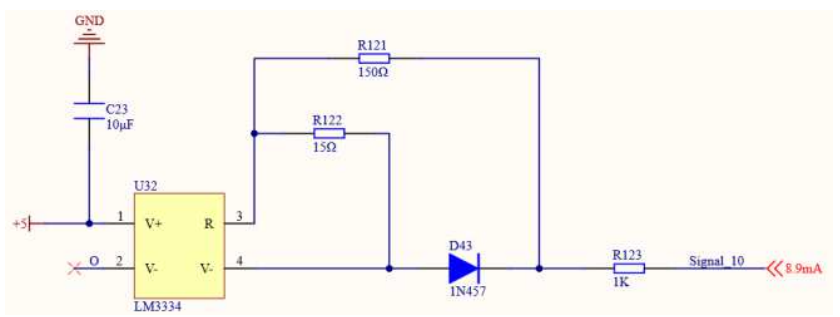

Figure 2 Constant current source power supply circuit diagram

After the above constant current source is powered, the sensor will output a dynamic signal with DC bi-as. In order to achieve direct coupling, attenuation and suppression of low-frequency signals, we design a two-stage active high-pass filter based on the integrated operational amplifier UA741CP. The specific circuit is shown in the Figure 3. The lower cut-off frequency of the high-pass filter circuit is determined to be $0.16 \mathrm{~Hz}$ by setting the values of the resistance and capacitance. The relationship between the cut-off frequency $f_{0 \mathrm{p}}$ and the resistance and capacitance are as shown in formula (1), $\mathrm{R}=100 \mathrm{k} \Omega$, $\mathrm{C}=0.1 \mu \mathrm{F}$, and simulation shows that this second-order active high-pass filter can effectively suppress low-frequency signals.

$$
f_{0 p}=\frac{1}{2 \pi R C}
$$

During the test, aliasing distortion may occur. We have added a two-stage active low-pass filter to the rear level of the second-order active high-pass filter to filter the interference of high-frequency signals and play a role in impedance matching [9].

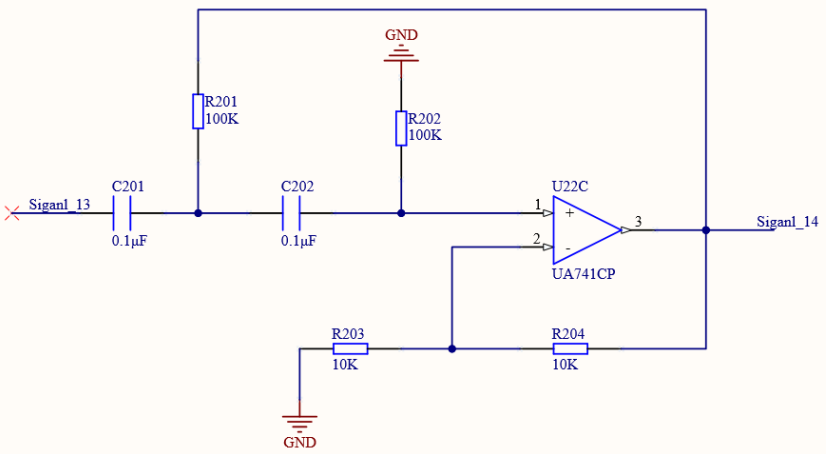

Figure 3 Second-order active high-pass filter circuit

Based on the integrated operational amplifier CMOS-TCL2274, the two-stage active Butterworth low-pass filter is designed [10], and the specific circuit of the Seren Keller circuit is shown in Figure 4. By set-ting the resistance value of $1000 \Omega$, the capacitance value of $1 \mathrm{nF}$, $m=11$, and $n=16$, the cutoff frequency of the filter is determined to be $12 \mathrm{kHz}$. From the amplitude corresponding graph, it can be seen that the func-tional relationship corresponding to the cut-off frequency is shown in equation (2).

$$
f_{0 c}=\frac{1}{2 \pi R C \sqrt{m n}}
$$

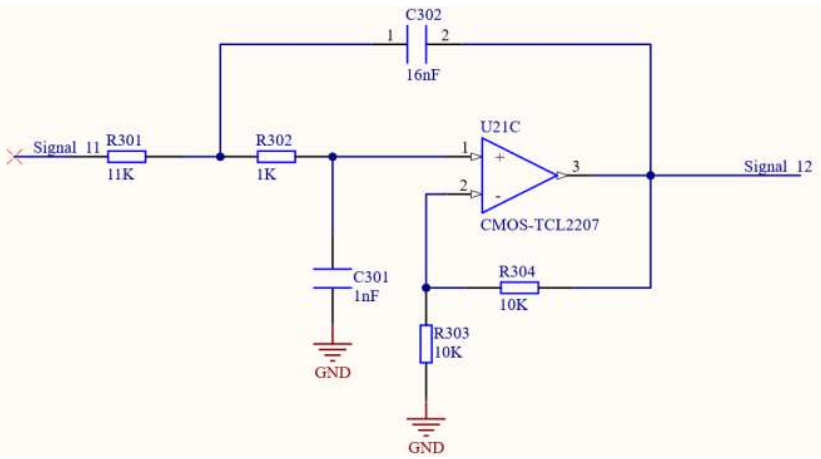

Figure 4 Two-stage active low-pass filter circuit

\subsubsection{A/D conversion module}

Onboard ADS1256 chip, 8-channel 24-bit high-precision ADC (4-channel differential input), 30ksps sampling rate. The controller block diagram of ADS1256 is shown as Figure 5:[11-14]

The working process of the module is as follows: ADC controller waits for the arrival of the trigger signal when the power is on; when the trigger signal is valid, the controller sets the chip select signal (CS) of channel 2, the timing preparation signal DRDY to low, and starts a collection and conversion process; At the same time the counter starts counting for 20s, and after the technology ends, it enters the next waiting trigger.[14-17] 


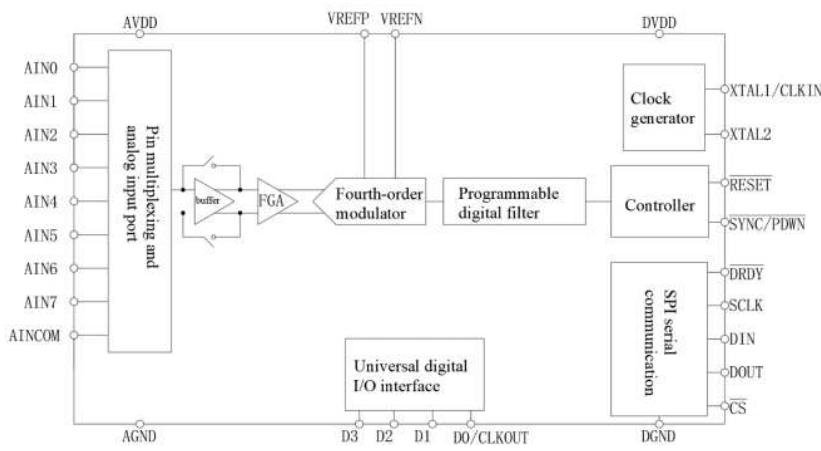

Figure 5 ADC controller frame

\section{2 software design}

The software design of this system is mainly aimed at $\mathrm{C}$ language programming under embedded Linux system. Raspberry Pi 4B has a built-in 4G DDR4 and a 16G SD card. In order to meet the requirements of high-speed and high-precision data recovery, real-time caching needs to be considered [18-19].

C programming is compiled with GCC compiler, combined with the WiringPi library that comes with the Raspberry $\mathrm{Pi}$, by querying the corresponding physical address of ADS1256, and using the SPI protocol register manual to realize the encapsulation of the corresponding data reading function.[20-23] Then turn on the SPI bus interface of the Raspberry $\mathrm{Pi}$, configure the corresponding driver, and then turn on the data collection of channels 1 , and then wait for the data of channel 1 to change. When the data is less than a specific value, start to collect the data of the acceleration sensor module, that is, the data of channel two is collected into the designated buffer space, and then the data in DDR4 is transferred to the SD card by means of polling buffer. The software flow is shown in Figure 6. [24-25] After the storage is over, the upper computer software is used to complete the recovery of the data through Gigabit Ethernet. In order to avoid the problem of data loss caused by battery power failure at the moment of water entering, we choose the polling cache method to achieve data caching. The so-called polling cache storage method is to open up a cache as large as possible in the internal RAM of the Raspberry Pi Space, after 1 timer period, start to transfer data to the SD card, wait until the storage space is full, overwrite the previous data, continue data filling, adjust the size of the buffer, so that the data collection and storage process will not be interrupted.

The sampling rate of ADC is 30ksps. The SPI of the Raspberry Pi can achieve a communication speed of up to $32 \mathrm{Mbps}$, meeting the transmission requirements of the ADC. We use the maximum conversion time of the ADC as a benchmark. It is set in the timer interrupt service routine to store the A/D conversion result in RAM buffer every $33.3 \mu \mathrm{s}$.

During the specific implementation, a BUF with a cache of $1000 \mathrm{~KB}$ was opened in the memory DDR4 of the Raspberry $\mathrm{Pi}$. When the sampling rate is controlled by the timer to be $100 \mathrm{kHz}, 1000 \mathrm{~KB}$ of data is stored in the buffer, and then cyclically, to realize the polling buffer, so as to avoid the problem that the data cannot be buffered in time when the power is cut off due to the impact of water.

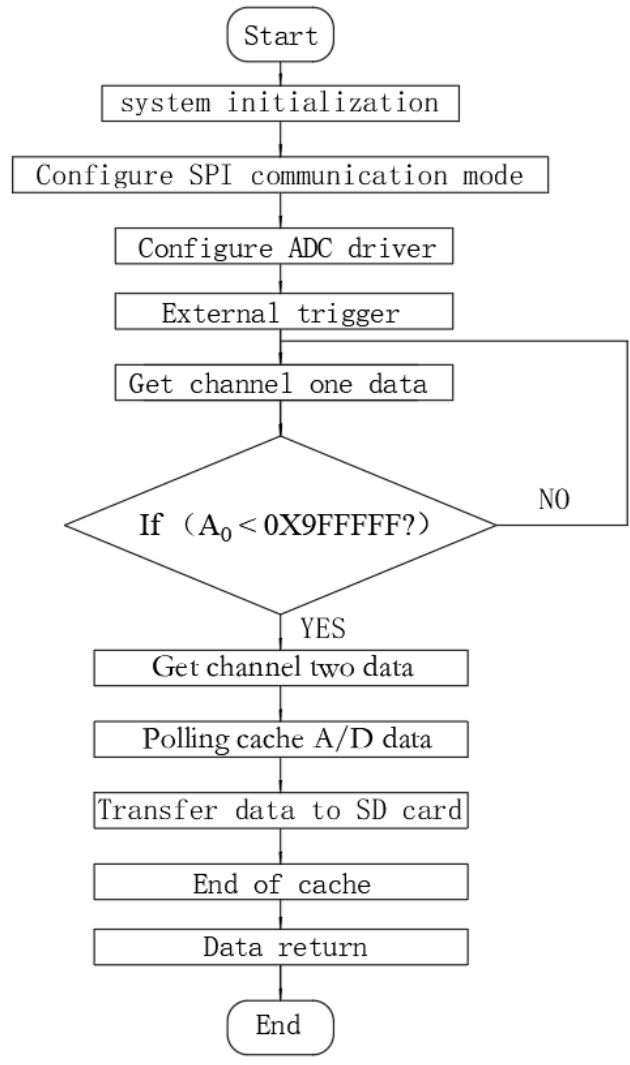

Figure 6 Program design process

\section{Verification of data acquisition device}

\subsection{Experimental design}

The encapsulated acceleration recovery device and the buffer head are fixedly connected by bolts to form a projectile impact head. The schematic diagram of the projectile impact head is shown in Figure 7.

This verification experiment uses two impact heads with different impact angles, and the processed physical map is shown in Figure 8.

Since the pool cannot be used to observe the impact process of high-speed water entry, we established an alternative experimental device assembled as shown in Figure 9. Projectiles equipped with acceleration cap-ture cards are shot into the water tank. The initial velocity and water entry attitude are recorded by laser beam and 
high-speed camera to ensure that the acceleration during the water entry impact process is consistent with the simulation attitude. The projectile is fired from a standard artillery device aimed at the center of the tank from the top of the tank.

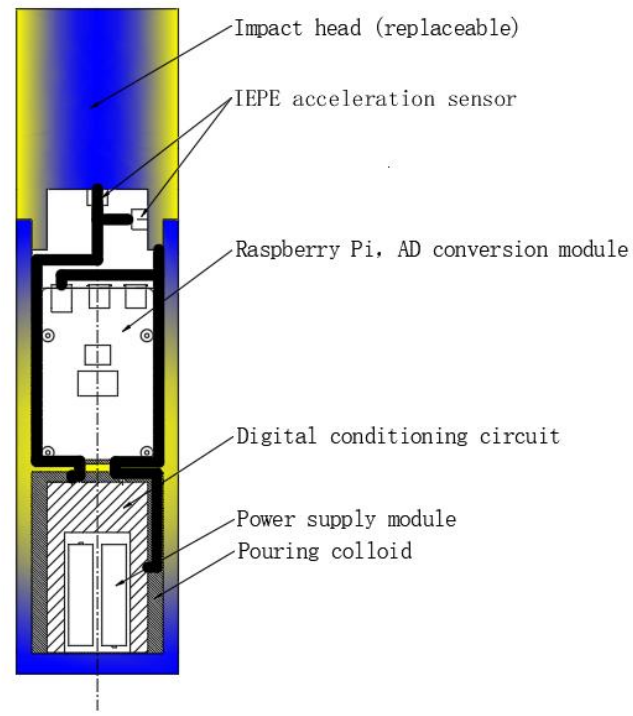

Figure 7 The internal structure of the projectile impact head

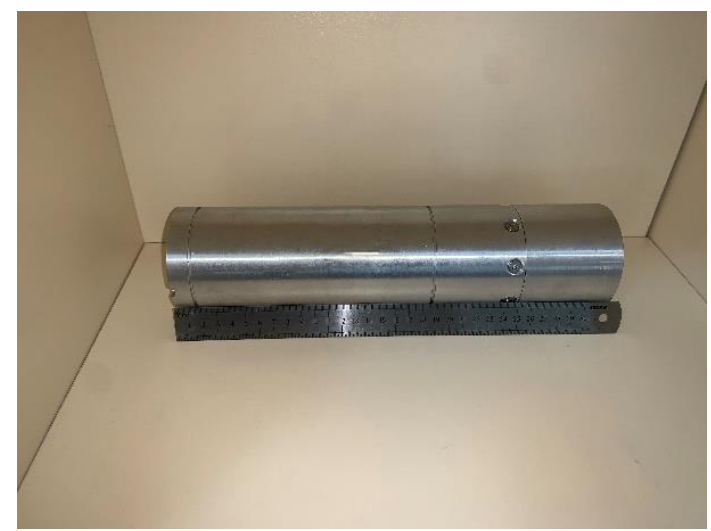

Figure $80^{\circ}$ impact head

Adjust the acceleration and initial velocity of the projectile by adjusting the mass of the propellant in the cartridge. It is expected that the initial velocity of the projectile at the moment of entering the water is about 100 $\mathrm{m} / \mathrm{s}$, which is used to compare the impact process generated by the simulation. The posture is straight down, eliminating the uncontrollable influence caused by the trigger line, temperature and vibration. Phan-tom color high-speed cameras are deployed to record the initial velocity of the impact and the posture of the projectile entering the water. The selected frame rate is 10000 frames per second, that is, one frame is taken every $100 \mu$ s. These settings are selected based on the early numerical simulation results of LS-DYNA. After the test, the acceleration during the water impact can be obtained.

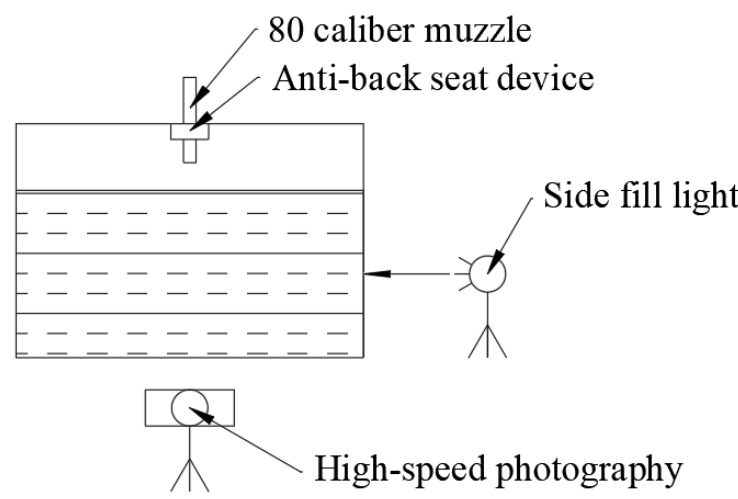

Figure 9 Schematic diagram of impact tank structure

In this test, $0^{\circ}$ flat head and $45^{\circ}$ oblique head projectiles were used for vertical shooting test. Intercept the corresponding impact fragments, and get the $0^{\circ}$ flat head water impact process as shown in Figure 10:

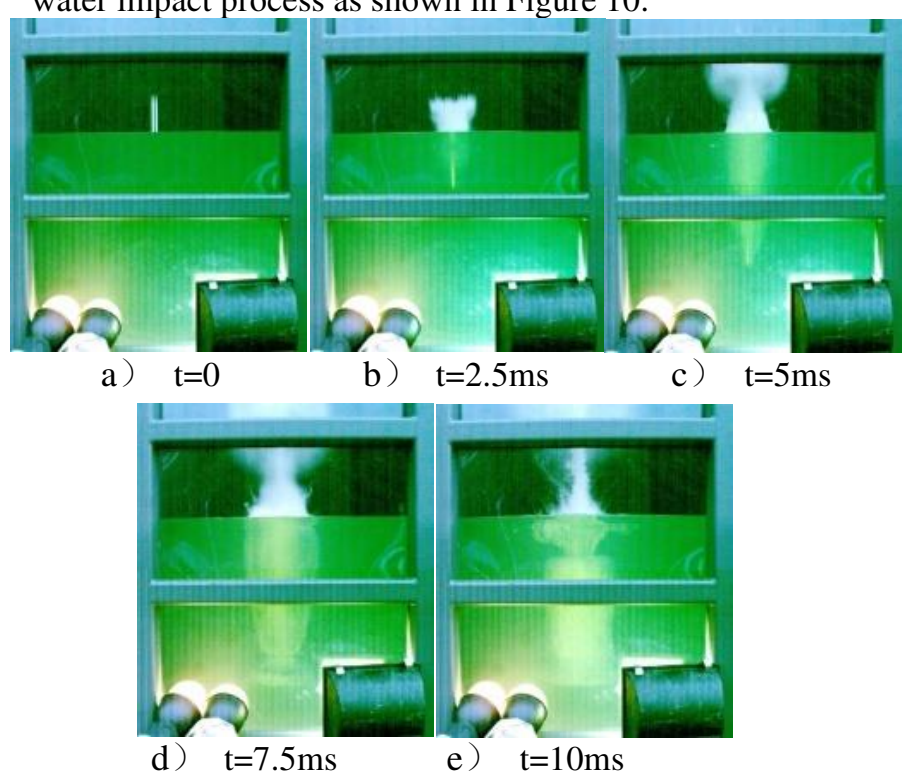

Figure10 The vertical water penetration process of a $0^{\circ}$ flat-headed projectile

\subsection{Simulation data analysis}

In order to study the force distribution of the projectile under different entry angles when entering the water, LS-Dyna was used for dynamic simulation analysis and a 3D model was established [26]. The projectile body, protective material, and water area are established separately. The air has little effect on the maxi-mum impact force, so the air area is ignored in this modelling. Because the structure is symmetrical in this experiment, a quarter model is used for modelling, and the element system is $\mathrm{cm}$-sg. A quarter rigid cylinder with the same 
mass as the test projectile is established to simulate the projectile, its mass and thickness the $2.5 \mathrm{~cm}$ test piece is equivalent, and the side of the cube water is $60 \mathrm{~cm}$. A non-reflective surface is established on the asymmetric surface of the water area and the ground of the water area to simulate the situation of an infinite water area [27]. The model view is shown in Figure 12.

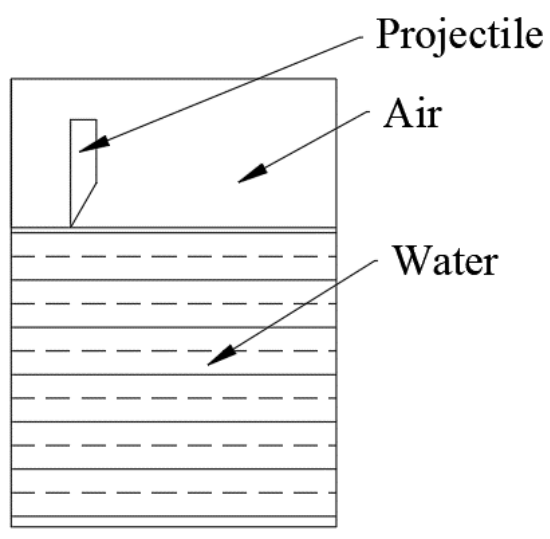

Figure 12 Schematic diagram of simulation model

The grid division is shown in Figure 13. Because of the study of the water stress and overload, the La-grangian grid is used for the water entering, and the ALE grid is used for the calculation of the water.

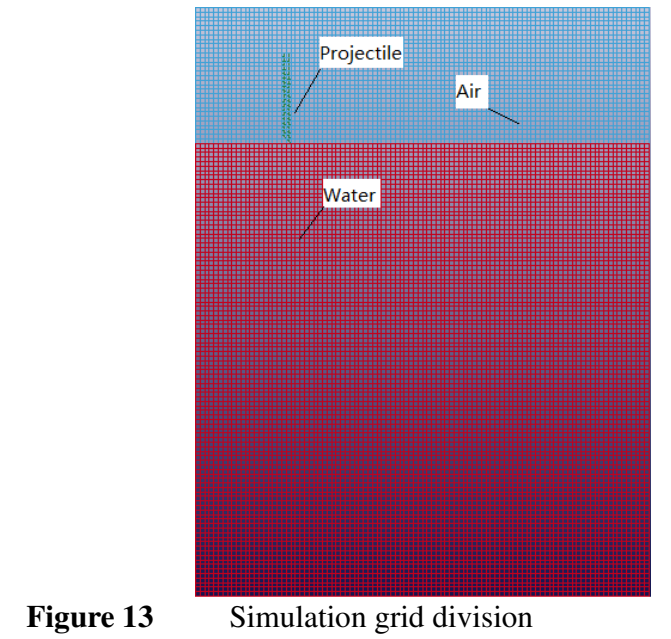

\subsection{Analysis of data results}

Use Excel to integrate the experimental data and simulation data into a table. From the scatter diagram, it can be seen that there is a certain difference between the two. Due to the small collection time interval of the experimental data, there will be a certain error in the data time.

In order to quantitatively analyse the difference between experimental measurement results and simulation results, specific error evaluation indicators are required. According to past experience, the accuracy of the parameters can be evaluated by using errors. Since in the water impact experiment, the speed is $100 \mathrm{~m} / \mathrm{s}-0 \mathrm{~m} / \mathrm{s}$, which is the acceleration attenuation curve, the square error, the average maximum relative error, and the average peak error. The performance of the data recovery device system is of little significance, so when considering two waveforms, we only need to evaluate the energy difference of the waveform, which is to introduce the square sum error to describe the energy difference between the simulated signal and the experimental measurement signal [28].

$$
E_{r s p}=\frac{\sum_{i=1}^{N}(X[i])^{2}+\sum_{i=1}^{N}(Y[i])^{2}}{\sum_{i=1}^{N}(Y[i])^{2}}
$$

In Equation 3, it can be known that $\mathrm{X}$ [i] represents the acceleration signal obtained from the experimental data, $\mathrm{Y}$ [i] represents the actual acceleration signal (using approximate simulation data as the ideal measured acceleration data), and $\mathrm{N}$ represents the sampling point.

According to cluster analysis, the correlation between experimental data and simulation data is determined.

Correlation coefficient is the earliest statistical indicator designed by statistician Carl Pearson. It is a measure of the degree of linear correlation between variables. Due to different research objects, correlation coefficient can be used to reflect the close degree of correlation between variables [29].

The definition is as follows:

$$
r(X, Y)=\frac{\operatorname{Cov}(X, Y)}{\sqrt{\operatorname{Var}[X] \operatorname{Var}[Y]}}
$$

Use MATLAB to import the experimental data generated by the experiment and simulation, and use the function to verify the correlation of the two-acceleration data. It can be seen that: $\mathrm{A} 1=[1,0.6974,0.6974,1]$, at this time $r<0.8$, we can see that the correlation is weak. Analysis The reason is that the division standard of the data time point has a great influence on the correlation. The data between the extreme values is intercepted by sections, and MATLAB is used again for corresponding analysis. The intercepted data are shown in Table 2 .

At this time, A1= [1,0.9973,0.9973,1] can be obtained. At this time, $r<<0.8$, it can be seen that the two are highly correlated, indicating the consistency of the experimental data and the simulation data.

In order to compare the changes and trends of the two sets of data more clearly, we use Origin to draw the experimental data and simulation data on a line chart at the 
Table 2 Intercepted data fragments

\begin{tabular}{|c|c|c|c|c|c|c|c|}
\hline & 1 & 2 & 3 & 4 & 5 & 6 & 7 \\
\hline Sim data 1 & 13062.84 & 10296.69 & 6706.22 & 5425.56 & 5346.87 & 4308.31 & 2769.63 \\
\hline Exp data 1 & 0X00334C & 0X00285E & 0X001AA7 & 0X0015CA & 0X00154E & 0X001143 & 0X000B28 \\
\hline Sim data 2 & 3342.70 & 3368.65 & 2257.96 & 2590.11 & 2093.34 & 1520.12 & 1988.96 \\
\hline Exp data 2 & 0X00334C & 0X00285E & 0X001AA7 & 0X0015CA & 0X00154E & 0X001143 & 0X000B 28 \\
\hline Sim data 3 & 1290.30 & 996.24 & 1063.01 & 676.71 & 664.40 & 421.26 & 533.37 \\
\hline Exp data 3 & $0 \times 000574$ & 0X00043E & 0X000481 & $0 \mathrm{X} 0002 \mathrm{~F} 4$ & $0 \mathrm{X} 0002 \mathrm{~F} 4$ & $0 \times 000260$ & $0 \times 000265$ \\
\hline Sim data 4 & 275.10 & -50.73 & -23.91 & -204.42 & -183.50 & -376.87 & -530.23 \\
\hline Exp data 4 & 0X00016D & 0X80000C & 0X000026 & $0 \times 800076$ & $0 \times 800060$ & $0 \times 800126$ & 0X8001D8 \\
\hline Sim data 5 & -466.12 & -629.45 & -670.71 & -566.47 & -712.48 & -793.03 & -747.14 \\
\hline Exp data 5 & 0X800181 & $0 X 800216$ & 0X80024D & 0X8001D2 & $0 \times 800264$ & 0X8002B5 & 0X800287 \\
\hline
\end{tabular}

same time, at this time, the relationship between the two can be explicitly expressed.

It can be found through the line graph that the changes and trends of the experimental data and the simulation data are basically the same. Figures 14 and 15 are respectively the trend graphs of the acceleration change in the horizontal and vertical directions when the water enters at $45^{\circ}$.

To sum up, compare the shock acceleration obtained by experiment and simulation. Since the data generated by the simulation is that the acquisition frequency is $100 \mu \mathrm{s}$, and the $\mathrm{A} / \mathrm{D}$ conversion module used is $83 \mu \mathrm{s}$ and $100 \mu \mathrm{s}$, there is a certain error in the calibration of the initial time setting. Within the allowable range of error, it can be found that the experimental and simulation results are consistent, which verifies the performance of the device.

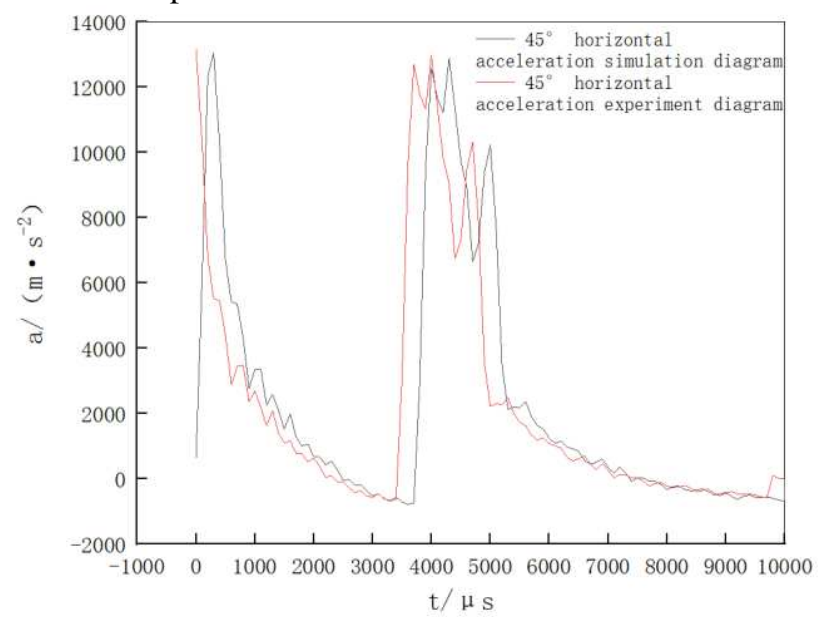

Figure 14 Comparison chart of horizontal acceleration of $45^{\circ}$ entering water

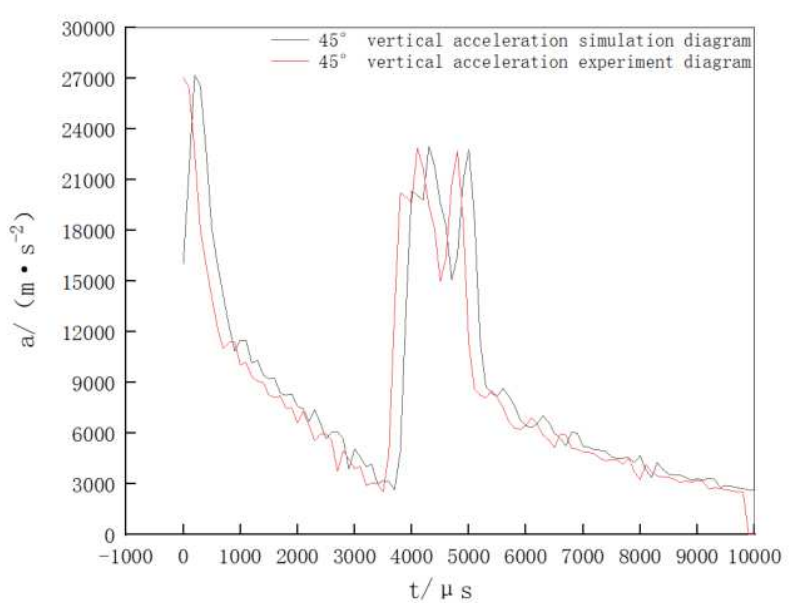

Figure 15 Comparison chart of vertical acceleration of $45^{\circ}$ water entry

\section{Conclusions}

Aiming at the high-speed projectile water impact recovery test system, using real-time storage technology and combining the actual object and layout environment, the design of a dedicated volume and anti-overload storage test device was completed. The test device uses a high anti-noise IEPE sensor. The test device is designed with a stable constant current source circuit and a second-order active filter, which will not cause signal energy loss in the passband, and the two-stage connection has little mutual influence.

Use high-precision A/D (ADS1256) chip to achieve analog-to-digital conversion, The data communication between the main control unit and the ADC is realized through the serial peripheral interface SPI, and the high-speed data transmission is completed, and the transmission data is stable. The main control unit stores the collected digital signals in the corresponding memory card through a new data buffer technology.

In the software design, the disconnection signal is used as the trigger signal, which can effectively avoid the false 
triggering phenomenon during the test. In terms of data caching, a real-time polling caching scheme is designed to solve the problem of partial data loss caused by power outages in a specific water impact environment. Finally, the use of mathematical statistics to compare and analyze the energy difference of the waveform between the experimental data and the simulation data shows that: There is no obvious difference between the waveform energy of the simulation data and the experimental results, the overall deceleration trend is basically the same, and the mathematical analysis results show that the two data are consistent; There is no data loss, that is, there is no frame error and frame loss in the transmitted data. It can be concluded that the data recovery device can provide reliable basic data support for the mechanics research and parameter setting of related fields of water impact. However, this design is a recovery test device designed in a specific test state, using Raspberry Pi as the core capture card, without considering the internal high-speed storage hardware design of the system, and further overall research is needed.

\section{Declaration}

\section{Acknowledgements}

The authors sincerely thanks to Professor Zhi-Yu Shao of Beijing Institute of Technology University for his critical discussion and reading during manuscript preparation.

\section{Funding}

Not applicable

\section{Availability of data and materials}

The datasets supporting the conclusions of this article are included within the article.

\section{Authors' contributions}

The author' contributions are as follows: Shuai Zhang was in charge of the whole trial; Shuai Zhang wrote the manuscript; Zhi-Yu Shao assisted with sampling and laboratory analyses.

\section{Competing interests}

The authors declare no competing financial interests.

\section{Consent for publication}

Not applicable

\section{Ethics approval and consent to participate}

Not applicable

\section{References}

[1] Golovinskiy, S. M. Kruchinin, A. S. Musatkin, M. M. Burakov and P. A. Gornostaev, "Technological Design of the MEMS-Accelerometer Sensor Element for Ultra-Large Acceleration Ranges," 2020 IEEE Conference of Russian Young Researchers in Electrical and Electronic Engineering (EIConRus), St. Petersburg and Moscow, Russia, 2020, pp. 2135-2138, doi: 10.1109/EIConRus49466.2020.9039038.

[2] Levinzon F. Fundamental Noise Limit of an IEPE Accelerometer[M]//Piezoelectric Accelerometers with Integral Electronics. Springer, Cham, 2015: 107-116.

[3] FANG Y Y,WU J,YANG K X,et al. Relationship between time constant and low frequency limit, phase difference of IEPE aceleration sensor and time constant measurement[J]. Electronic Measurement Technology,2020,43(09):177-181.

[4] ZHOU R Q,JIA Y F,PAN X B, et al. Design of Storage Test System for High Overload Signal of Gun Launching[J]. Measurement \& Control Technology,2017,36(004):43-47.

[5] LIAN Shuren, SHI Yunbo, ZHI Dan, et al. Design of Missile Borne Data Recovery System Based on Accelerometer[J]. Piezoelectrics \& Acoustooptics2017,39(2):316-320.

[6] LONG Z J,CHEN Y P,LIU J,CHEN X. Data Acquisition System Based on FPGA for Sewage Treatment Project[J]. Industrial Control Computer,2016,29(05):20-21.

[7] CUI Q,GU J L,LIU Q G,LUO H E,XIA Y. Design of high speed data collection system based on DSP+FPGA[J]. Foreign Electronic Measurement Technology,2020,39(01):93-98.

[8] ZHANG T,SONG P,GU X H,LIU Y L. Experimental Research on Vibration Effect of Confined Space with Input-gauge[J]. Explosive Materials,2011,40(01):9-11+15.

[9] WANG K,HAN L L. Network Data Acquisition Circuits Based on 7500P[J]. Microcontrollers Embedded Systems,2020,20(02):73-76.

[10]WU Z H,WU Z Q,WANG Y,ZHU X H. Acquisition and Storage Device of Shock Acceleration Signal[J]. Process Automation Instrumentation,2016,37(06):98-102.

[11]Hui Xin.Zhang,Zheng.Guo,Yong.Ye.Design of High-Speed Remote Image Data Storage Memory Based on LVDS[J].Advanced Materials Research,2013,2109(1212):1989-1993.

[12]Xue, Yuan,Song, Zhitang,Guo, Tianqi, et al.Al-Sb-Ge phase change material: A candidate for multilevel data storage with high-data retention and fast speed[J].Scripta materialia,2018,157:152-156.

[13]Jie Tao.Diao, Yi Nan.Wang,Zhao Lin.Sun, et al.Integrated Instrument of Ultra-High Speed Data Acquisition and Storage[J].Applied Mechanics and Materials,2012,1945(397):1126-1131.

[14]Min.Xie,Fu Hai.Zhao,Guo Pin.Tang.A Realization of Large Capacity, High-Speed Data Acquisition and Storage System[J].Applied Mechanics and Materials,2014,3252(1138):162-167.

[15]Yeong-Lin Lai,Wen-Jung Chiang.Characteristics of System in a Package of Synchronous Dynamic Random Access Memory for High-Speed Data Storage Applications[J].IEEE Transactions on Magnetics,2014,50(7 Pt.1):3501105-1-3501105-5.

[16] Yang.Jiao,Jun.Wang,Gang.He.Design on Measuring System of New Type Electrical Resistivity Imaging Exploring Ground Instrument[J].Key Engineering Materials,2014,3350(1240):369-374.

[17]Qu, Shao-Bo,Robert, Olivier,Lognonne, Philippe, et al.Low frequency noise elimination technique for 24-bit Sigma-Delta data acquisition systems[J].Review of Scientific 
Instruments,2015,86(3):034708-1-034708-8.

[18]Pereira R I S, Dupont I M, Carvalho P C M, et al. IoT embedded linux system based on Raspberry $\mathrm{Pi}$ applied to real-time cloud monitoring of a decentralized photovoltaic plant[J]. Measurement, 2018, 114: 286-297.

[19] Shinde P A, Mane Y B. Advanced vehicle monitoring and tracking system based on Raspberry $\mathrm{Pi}[\mathrm{C}] / / 2015$ IEEE 9th International Conference on Intelligent Systems and Control (ISCO). IEEE, Coimbatore, India ,2015: 1-6.

[20]Starynkevitch, Basile.MELT - a Translated Domain Specific Language Embedded in the GCC Compiler[J].,2011.

[21]Fursin, G.,Kashnikov, Y.,Memon, A.W., et al.Milepost GCC: Machine learning enabled self-tuning compiler[J].International Journal of Parallel Programming,2011,39(3):296-327.

[22]Basile Starynkevitch.MELT - a Translated Domain Specific Language Embedded in the GCC Compiler[J].,2011,(Proc.+DSL+2011):118.

[23]GCC2Verilog Compiler Toolset for Complete Translation of C Programming Language into Verilog HDL[J].ETRI Journal,2011,33(5):731-740. DOI:10.4218/etrij.11.0110.0654.

[24]Qi Shen.Zhu.Improving Program Performance via Auto-Vectorization of Loops with Conditional Statements with GCC Compiler Setting[J].Applied Mechanics and Materials,2013,2755(868):1410-1414.

[25]XIAOCHAO LI,DONGHUI GUO,BENBIN CHEN.Retargeting GCC Compiler for Specific Embedded System on Chips[J].WSEAS Transactions on Computers,2015,14:420-429.

[26]WANG Z,WU ML,DAI WL. Study on Load Characteristics of High-speed Water-entry of Large Caliber Projectile[J]. Journal of Ballistics,2020,32(1):15-22.

[27]Song Z J , Duan W Y , Xu G D , et al. Experimental and numerical study of the water entry of projectiles at high oblique entry speed[J]. Ocean Engineering, 2020, 211:107574.

[28]XU, Lei, et al. Design of Measurement Memory System Based on MEMS Acceleration Sensor. International Journal of Online and Biomedical Engineering (iJOE), 2018, 14.09: 110-123.

[29]Rodgers J L , Nicewander W A . Thirteen Ways to Look at the Correlation Coefficient[J]. American Statian, 1988, 42(1):59-66.

\section{Biographical notes}

Shuai Zhang, Shuai Zhang, born in 1996, is currently a Master candidate at State Key Laboratory of Explosion Science and Technology, Beijing Institute of Technology, China. His research interests include Mechatronics, mechanical testing.

Tel: 17801206564; E-mail: 2569119978@qq.com

Zhi-Yu Shao, born in 1977, is currently an engineer at, State Key Laboratory of Explosion Science and Technology, Beijing Institute of Technology, China. He received his $\mathrm{PhD}$ degree from Beijing University of Aeronautics and Astronautics, China, in 2007. His research interests include mechachonics engineering, man-machine system, robotics and ocean engineering.

Tel: 010-68911579; E-mail: shaozhiyu@ bit.edu.cn 
Figures

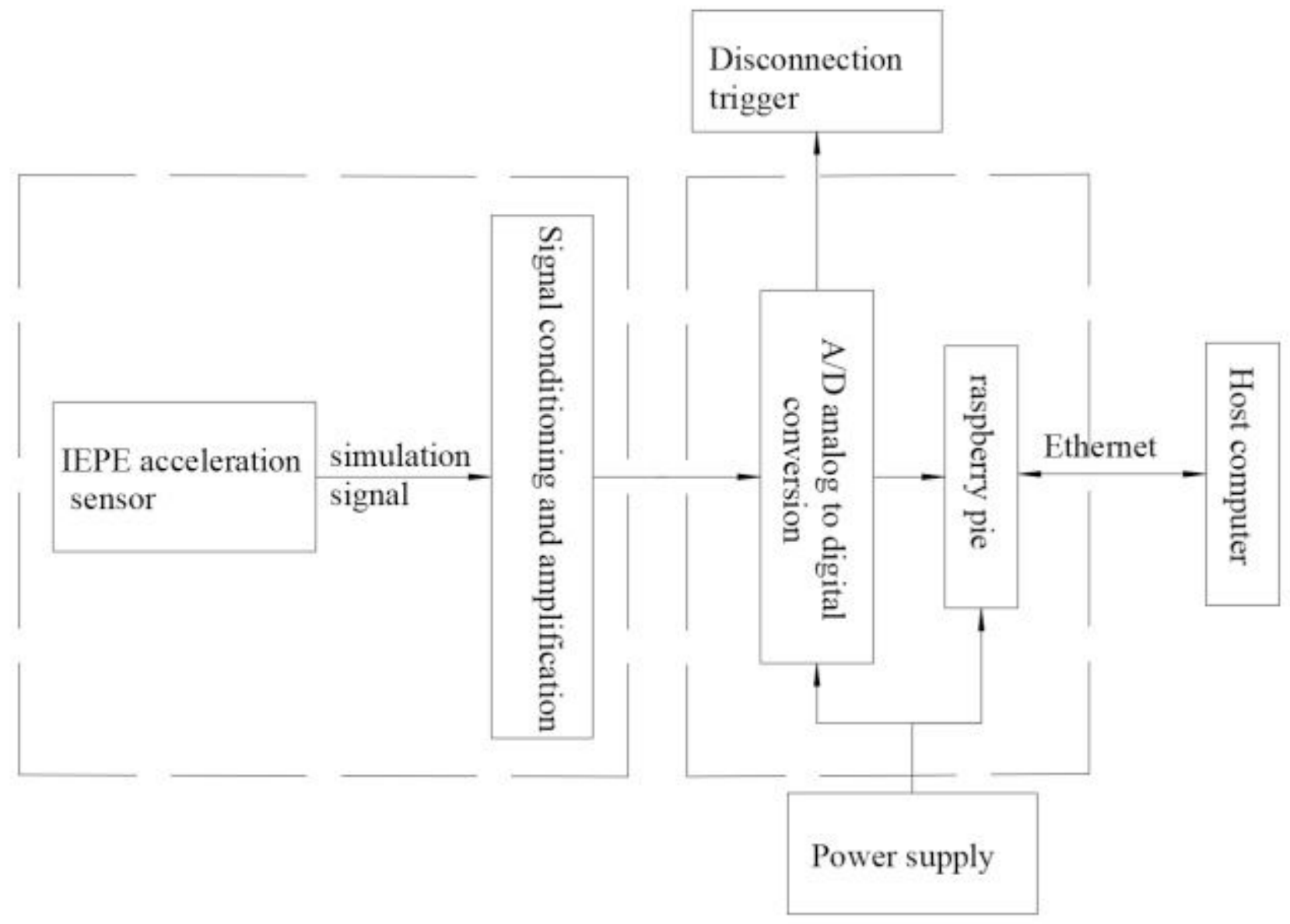

Figure 1

System structure block diagram.

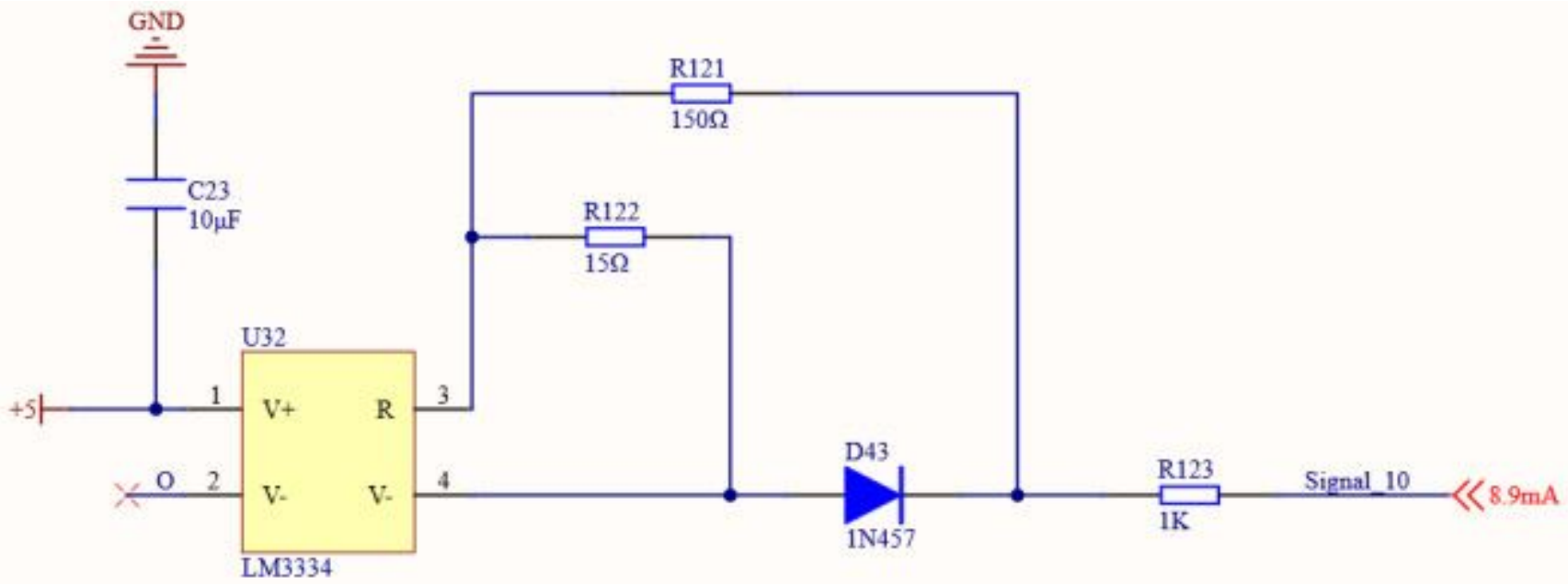

Figure 2

Constant current source power supply circuit diagram 


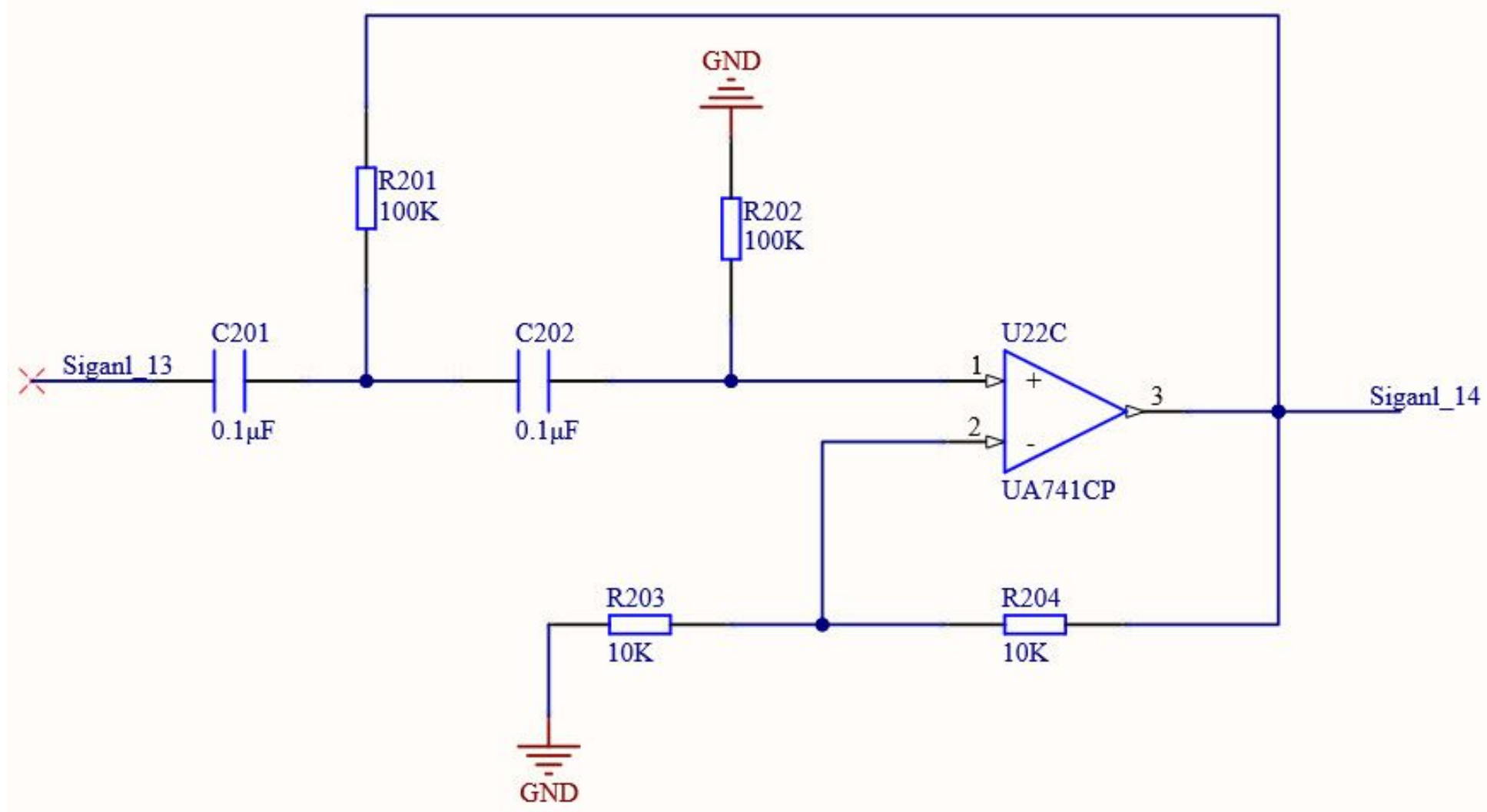

Figure 3

Second-order active high-pass filter circuit

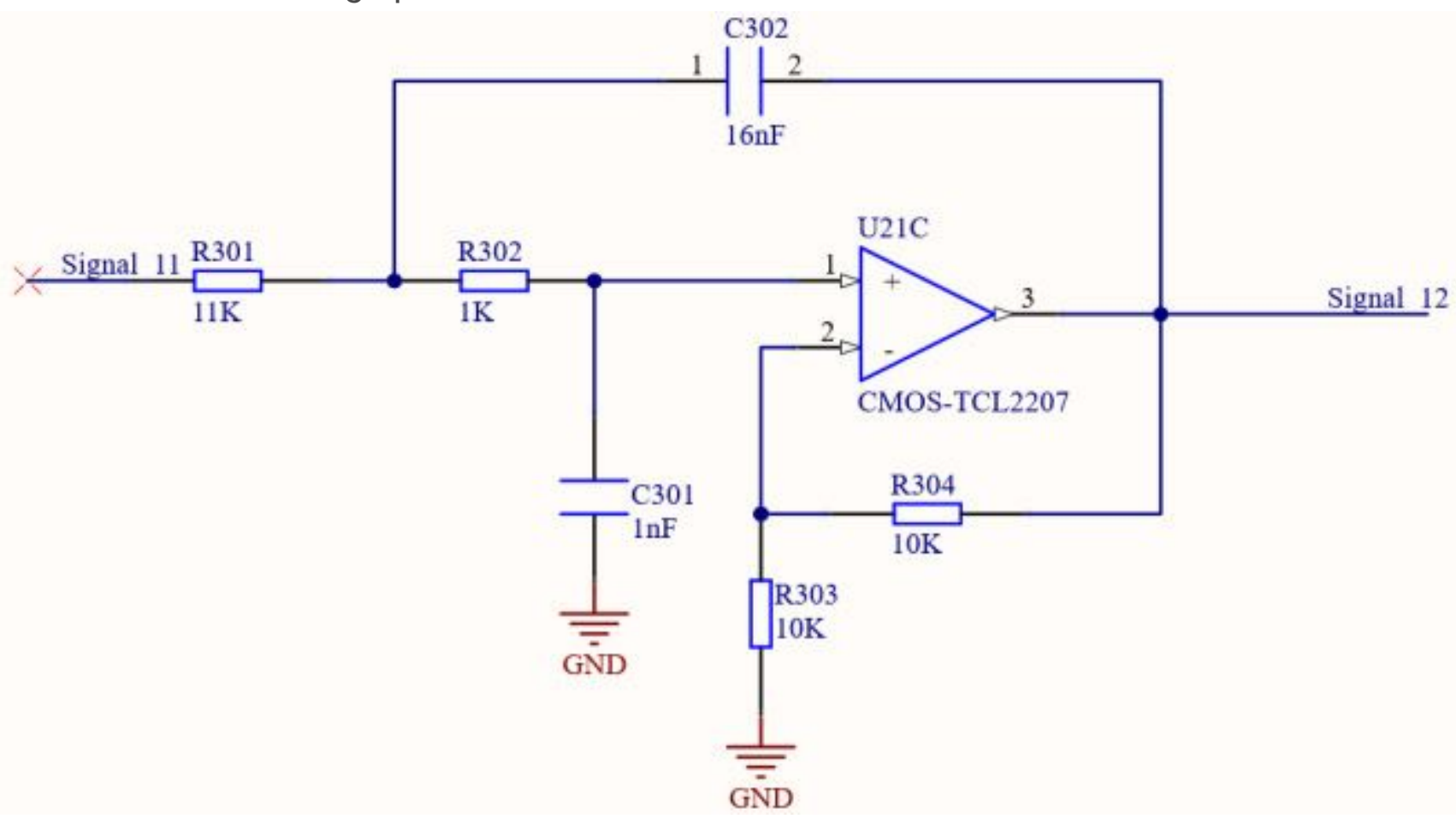

Figure 4

Two-stage active low-pass filter circuit 


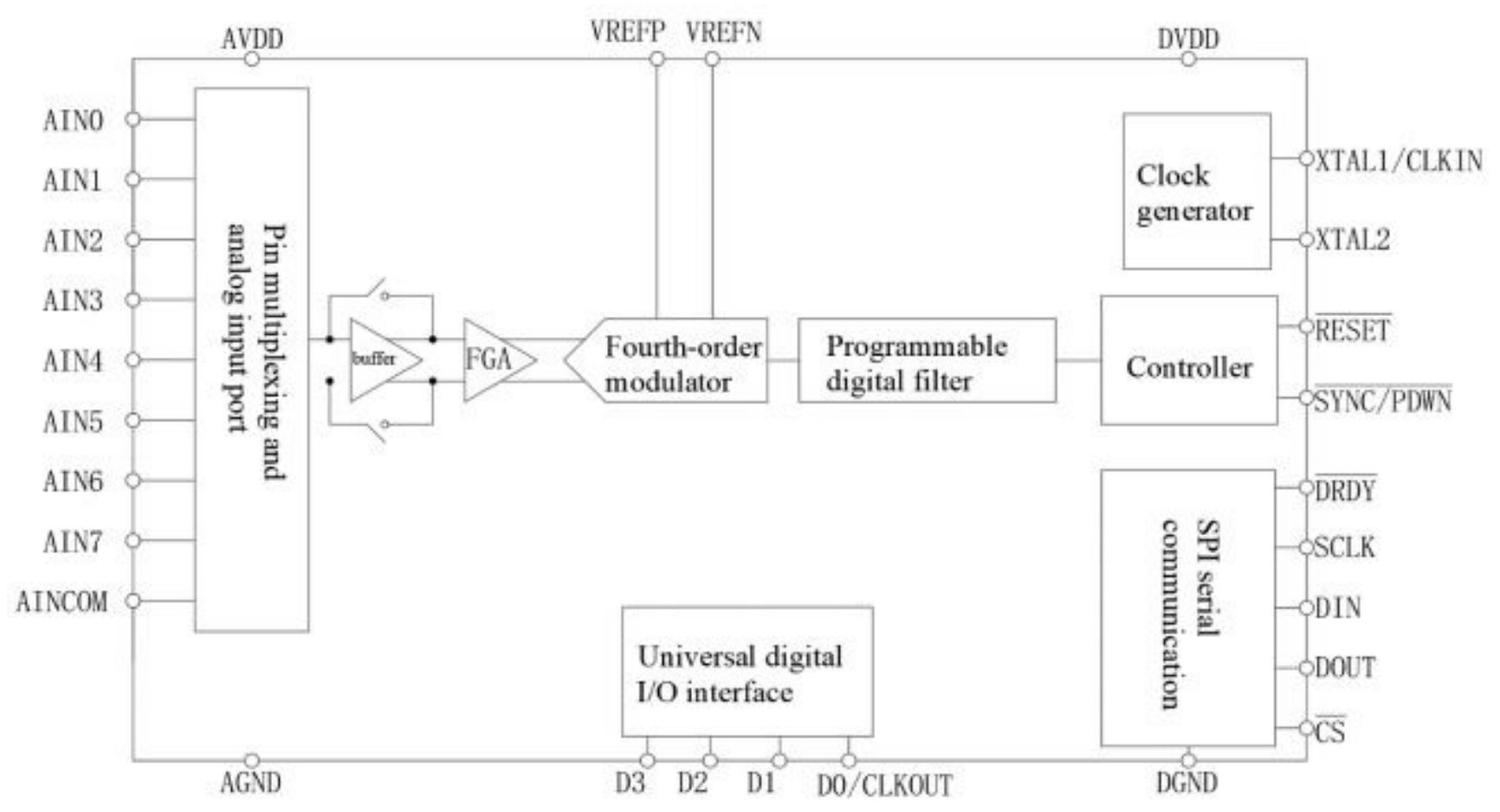

Figure 5

$A D C$ controller frame 


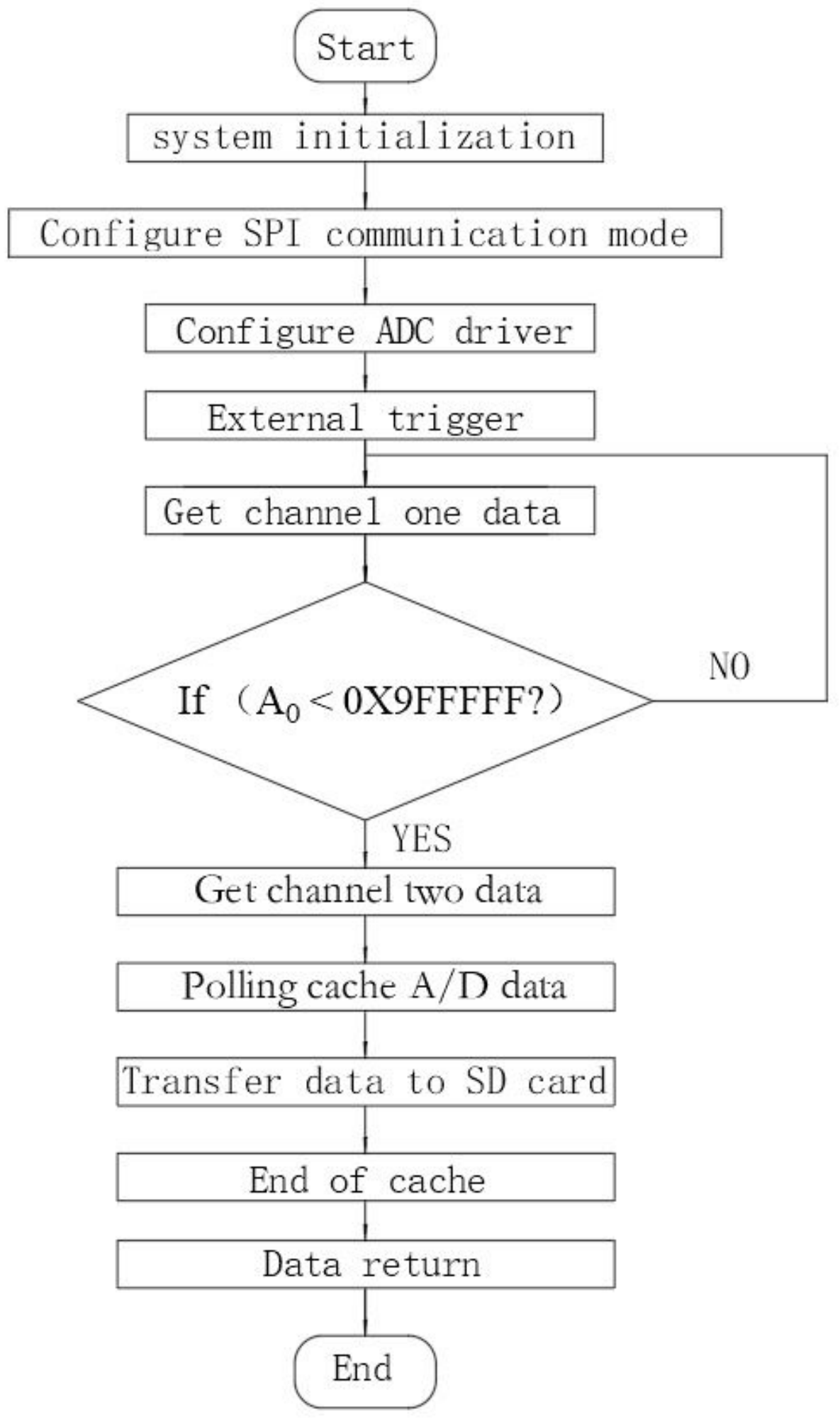

Figure 6

Program design process 


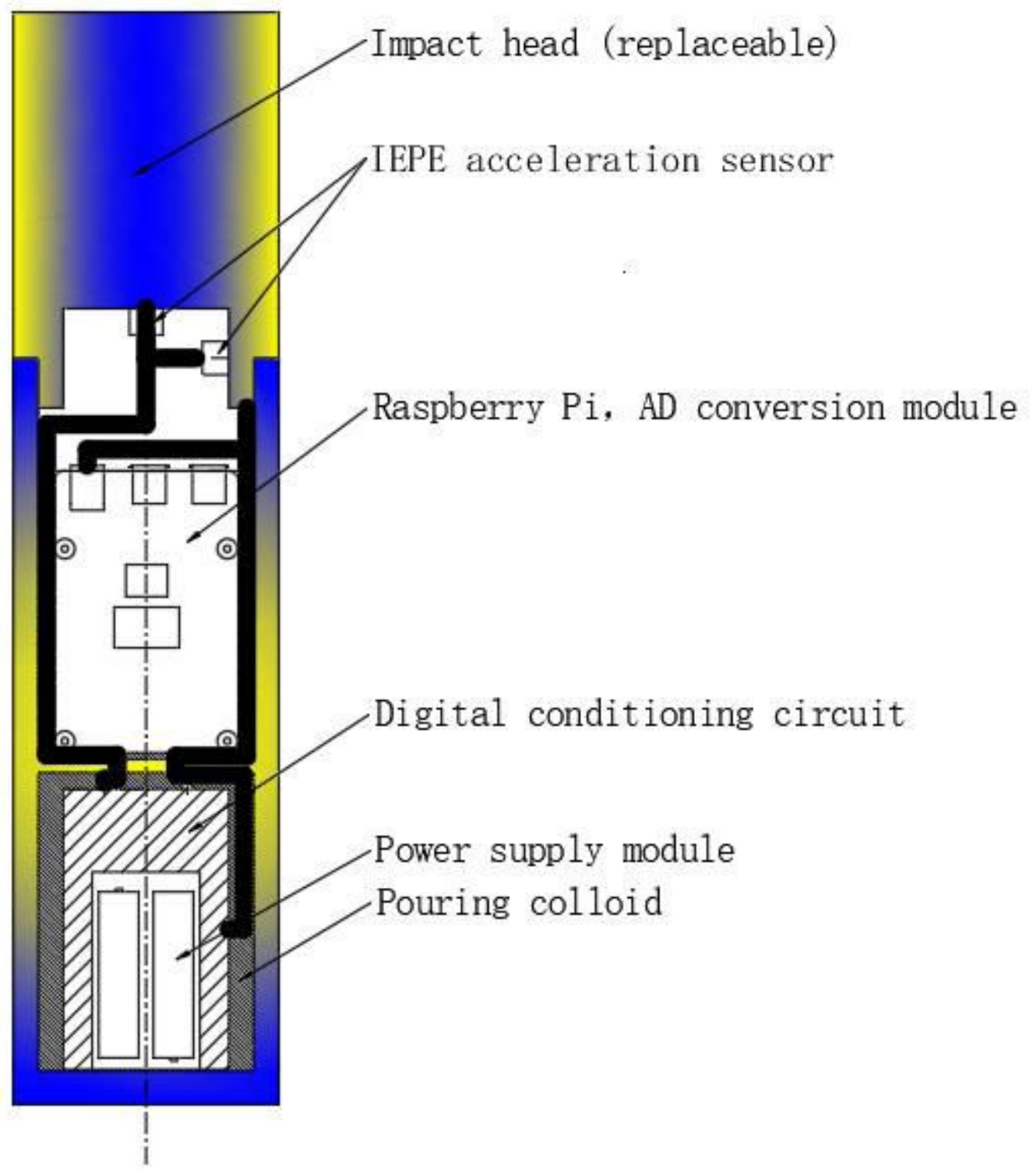

Figure 7

The internal structure of the projectile impact head 


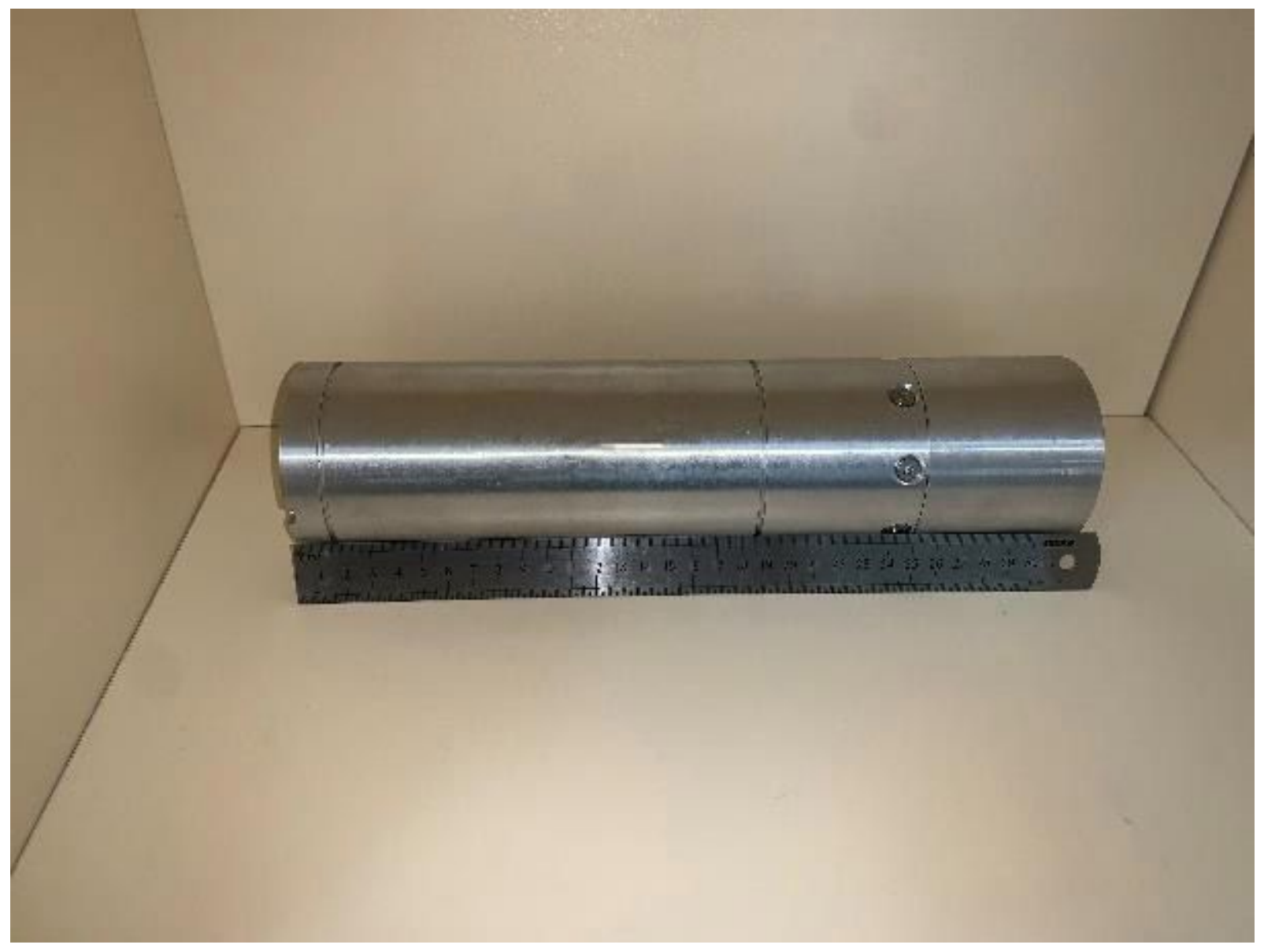

Figure 8

$0^{\circ}$ impact head 


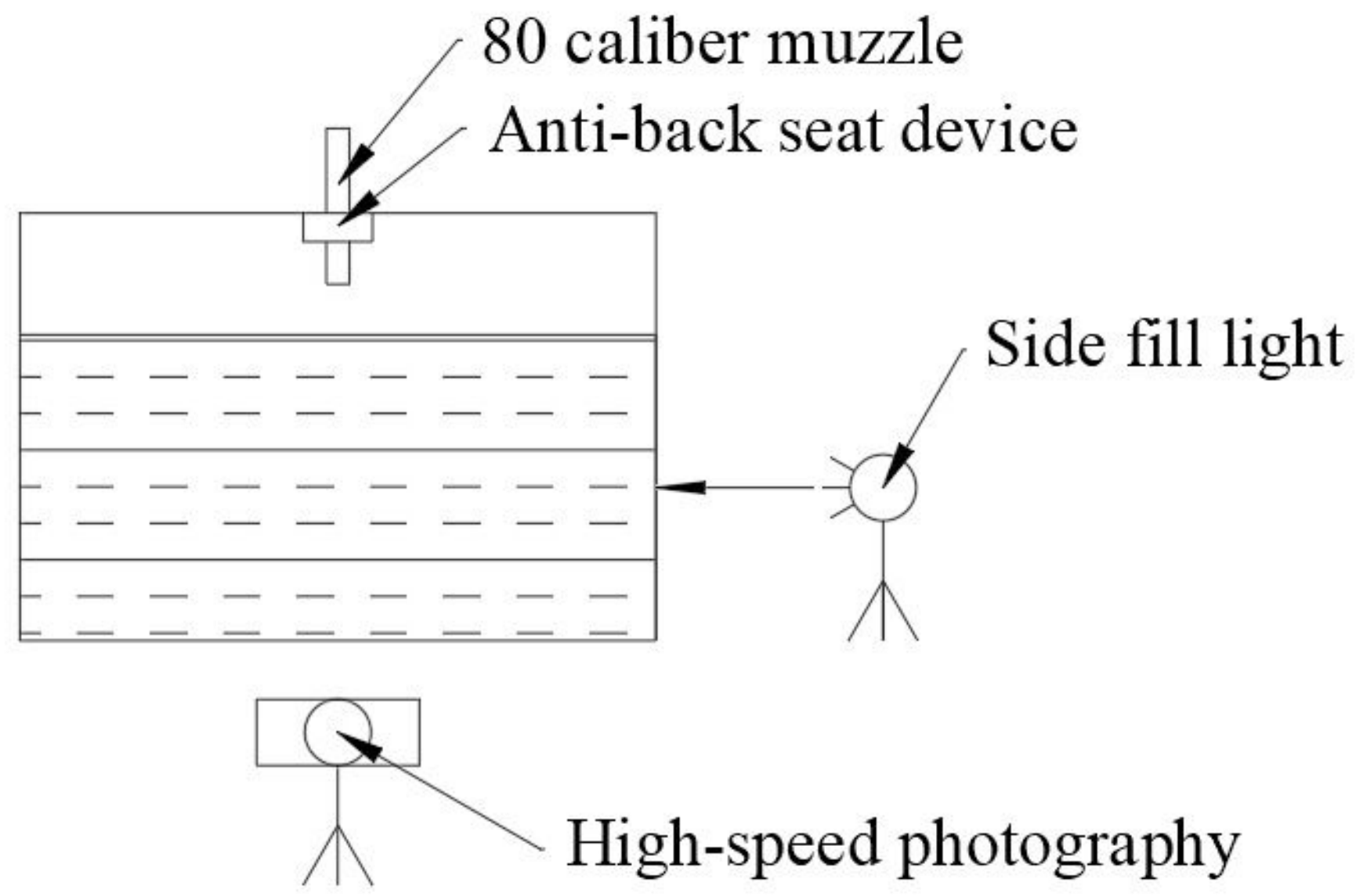

Figure 9

Schematic diagram of impact tank structure 


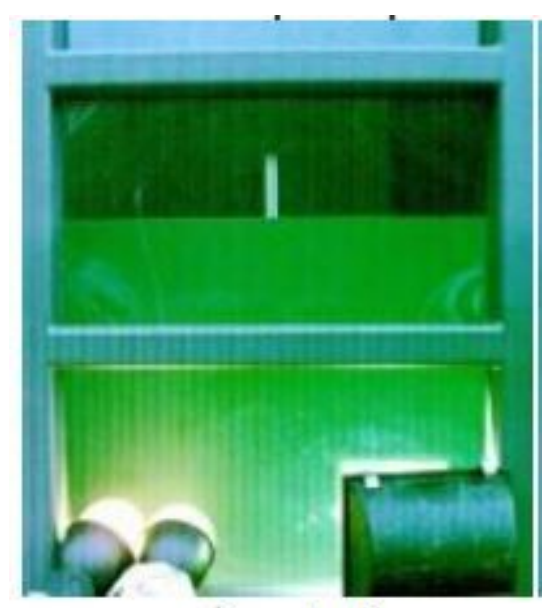

a) $\mathrm{t}=0$

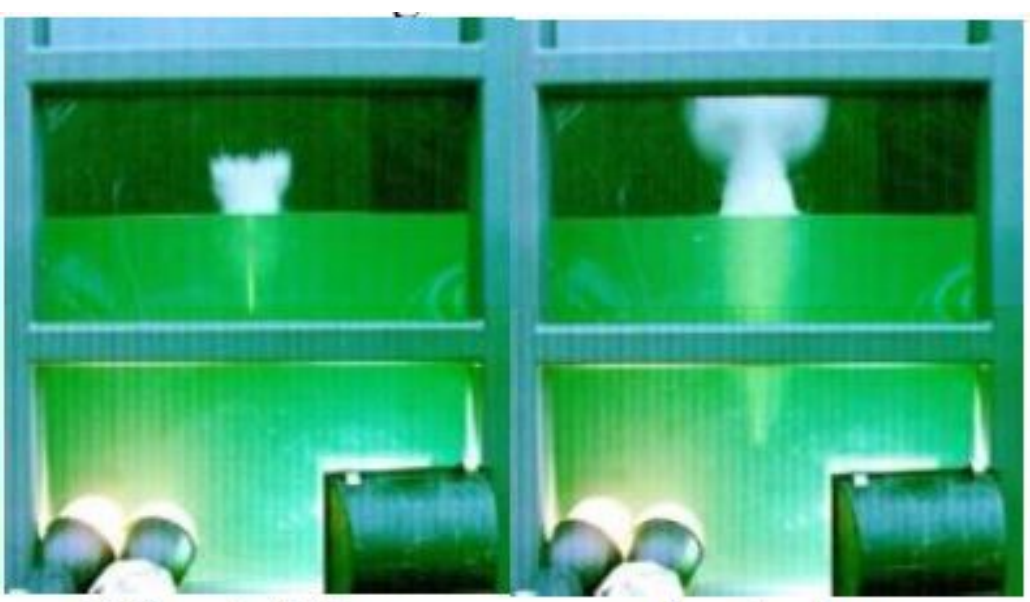

b) $t=2.5 \mathrm{~ms}$

c) $t=5 \mathrm{~ms}$

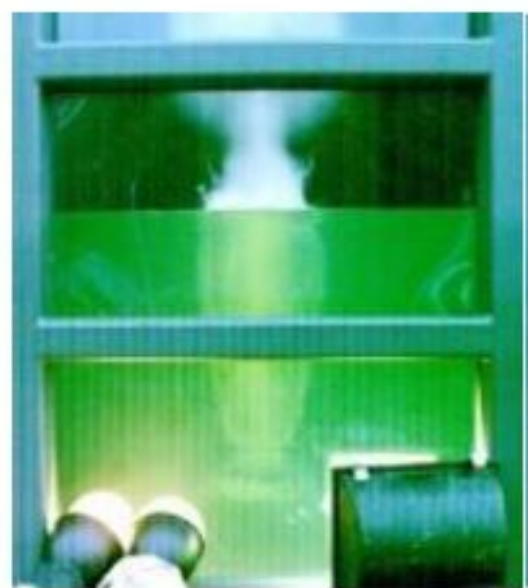

d) $\mathrm{t}=7.5 \mathrm{~ms}$

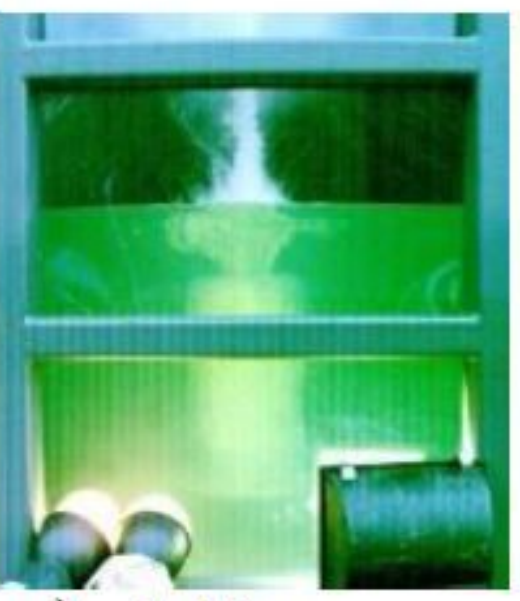

e) $\mathrm{t}=10 \mathrm{~ms}$

Figure 10

The vertical water penetration process of a $0^{\circ}$ flat-headed projectile

\section{Image not available with this version}

Figure 11 


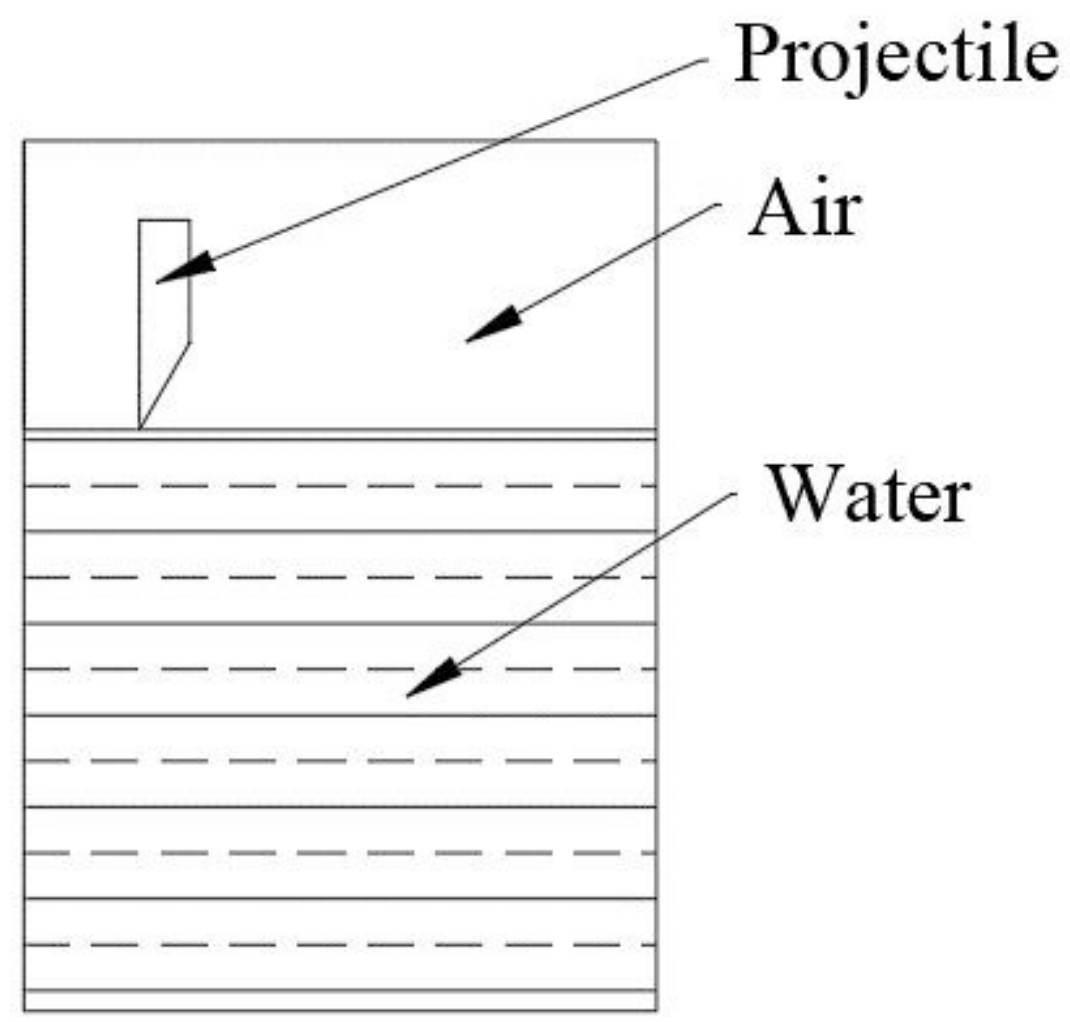

Figure 12

Schematic diagram of simulation model 


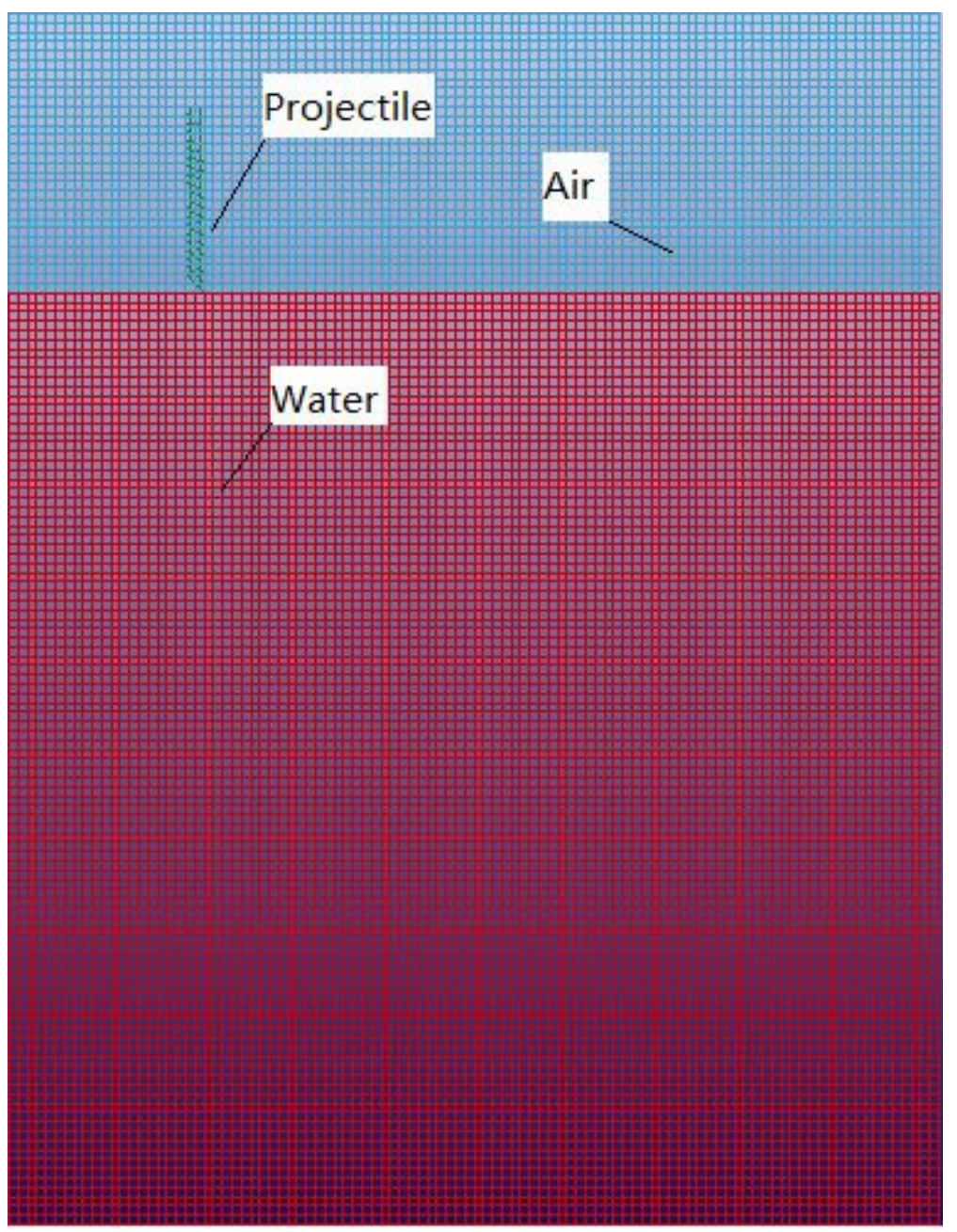

Figure 13

Simulation grid division 


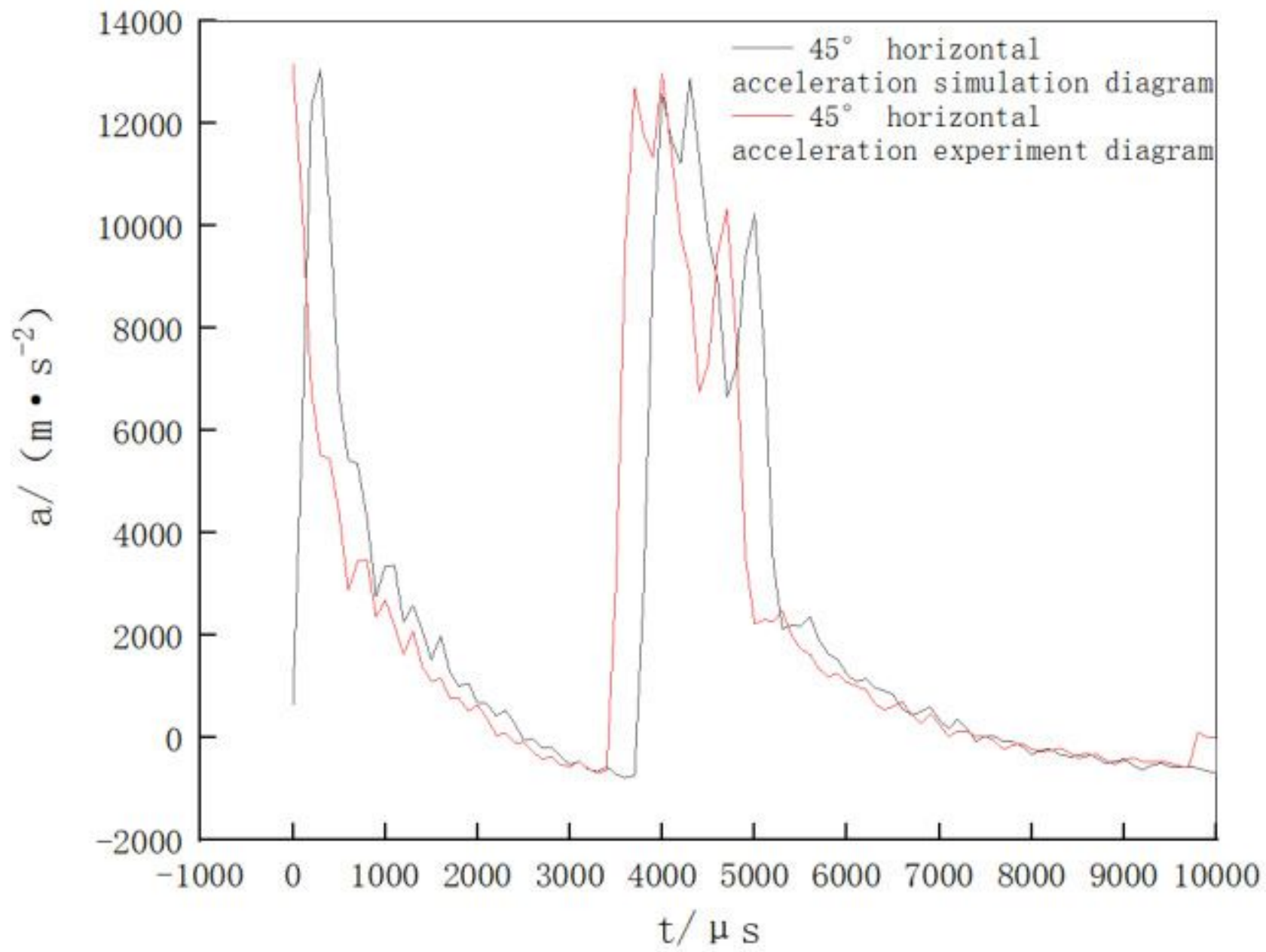

Figure 14

Comparison chart of horizontal acceleration of $45^{\circ}$ entering water 


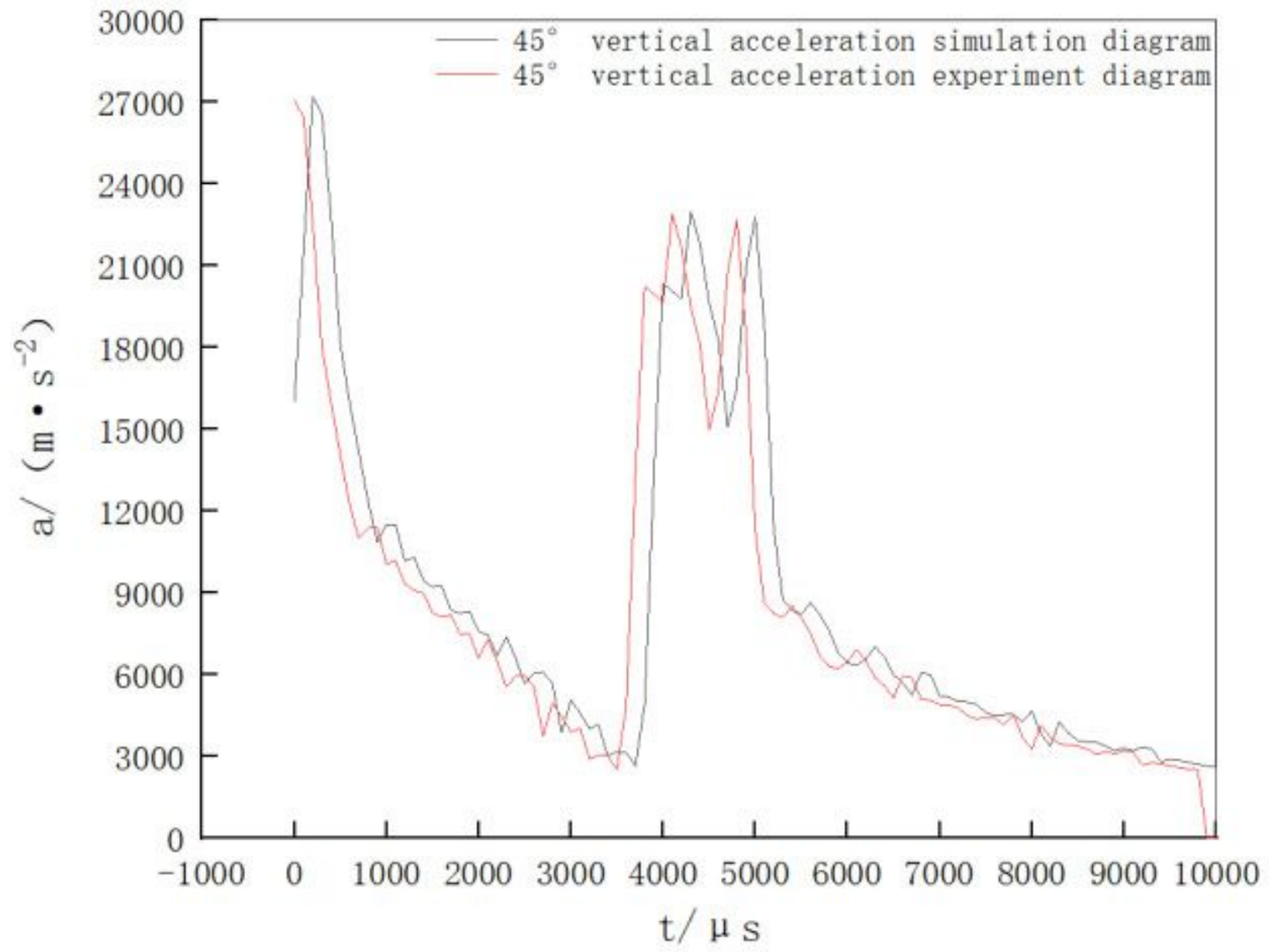

Figure 15

Comparison chart of vertical acceleration of $45^{\circ}$ water entry 